\begin{tabular}{|c|}
\hline $\begin{array}{r}\text { PRAMANA } \\
- \text { journal of } \\
\text { physics }\end{array}$ \\
\hline
\end{tabular}

\title{
A new observable to measure the top quark mass at hadron colliders
}

\author{
SIMONE ALIOLI ${ }^{1,2}$, JUAN FUSTER $^{3}$, ADRIAN IRLES $^{3, *}$, SVEN MOCH $^{2}$, \\ PETER UWER $^{4}$ and MARCEL VOS ${ }^{3}$ \\ ${ }^{1}$ Ernest Orlando Lawrence Berkeley National Laboratory, University of California, \\ Berkeley, CA 94720, USA \\ ${ }^{2}$ DESY, Platanenallee 6, 15738 Zeuthen, Germany \\ ${ }^{3}$ IFIC, Centre Mixte CSIC-Universitat de València, E-46071 València, Spain \\ ${ }^{4}$ Institut für Physik, Humboldt-Universität zu Berlin, Newtonstr. 15, 12489 Berlin, Germany \\ ${ }^{*}$ Corresponding author. E-mail: airqui@ific.uv.es
}

\begin{abstract}
The $t \bar{t}+1$-jet $+X$ differential cross-section in proton-proton collisions at $7 \mathrm{TeV}$ centre of mass energy is investigated with respect to its sensitivity to the top quark mass. The analysis includes higher order QCD corrections at NLO. The impact of the renormalization scale $\left(\mu_{\mathrm{R}}\right)$, the factorization $\left(\mu_{\mathrm{F}}\right)$ scale and of the choice of different proton's PDF (parton distribution function) has been evaluated. In this study it is concluded that differential jet rates offer a promising option for alternative mass measurements of the top quark, with theoretical uncertainties below $1 \mathrm{GeV}$.
\end{abstract}

Keywords. Top quark; mass; pole mass; NLO; cross-section; $t \bar{t}+$ jet; POWHEG; perturbative QCD.

PACS Nos 12.38.Bx; 14.65.Ha

\section{Introduction: $t \bar{t}+1$-jet $+X$ at NLO and definition of the observable}

Calculations for $t \bar{t}+1$-jet $+X$ final states were performed at NLO for hadronic collisions at both the Tevatron and the LHC [1]. Updated results of this calculation for the present LHC operating conditions at $7 \mathrm{TeV}$ are shown in table 1 which summarizes the results for calculations at LO and NLO accuracy in QCD considering different top quark pole masses. To estimate the impact of higher order corrections, the criteria used in ref. [1] were followed. The central results were obtained using $\mu \equiv \mu_{\mathrm{R}}=\mu_{\mathrm{F}}=m_{\mathrm{top}}^{p}\left(m_{\mathrm{top}}^{p}\right.$ being the pole top quark mass) and the CTEQ6.6 [2] PDF set. The scale dependences were being studied by varying the renormalization and factorization scales between $\mu=m_{\text {top }} / 2$ and $\mu=2 m_{\text {top }}$. Finally, the PDF dependences were studied by comparing the results obtained with the CTEQ6.6 and MSTW2008nlo90cl [3] PDF sets. 
Table 1. $t \bar{t}+1$-jet $+X$ cross-section for proton-proton collisions at $7 \mathrm{TeV}$ for different $m_{\text {top }}^{p}$. Jets were reconstructed using the FastJet Package [4] and the anti-Kt algorithm [5] with $R=0.4$. A selection cut of $p T$ (jet) $>50 \mathrm{GeV}$ and $|\eta|<2.5$ are applied to define the jet.

\begin{tabular}{lcc}
\hline$m_{\text {top }}^{p}$ & \multicolumn{2}{c}{$\sigma_{t \bar{t} j}$} \\
\cline { 2 - 3 }$(\mathrm{GeV})$ & LO & NLO \\
\hline 160 & $48.177(2)$ & $60.08(7)$ \\
165 & $41.739(2)$ & $52.19(6)$ \\
170 & $36.275(4)_{-12}^{+20}($ scale $) \pm 1(\mathrm{pdf})$ & $45.44(5)_{-6}^{+1}(\mathrm{scale}) \pm 1(\mathrm{pdf})$ \\
175 & $31.620(3)$ & $39.66(4)$ \\
180 & $27.641(1)$ & $34.69(4)$ \\
\hline
\end{tabular}

The observable we propose as alternative method to measure the top quark mass is defined by

$$
\frac{\mathrm{d} n_{3}}{\mathrm{~d} \rho_{\mathrm{s}}}\left(m_{\mathrm{top}}^{p}, \mu, \rho_{\mathrm{s}}\right)=\frac{1}{\sigma_{t \bar{t} j}} \frac{\mathrm{d} \sigma_{t \bar{t} j}}{\mathrm{~d} \rho_{\mathrm{s}}}\left(m_{\mathrm{top}}^{p}, \mu, \rho_{\mathrm{s}}\right),
$$

where $\sigma_{t \bar{t} j}$ denotes the cross-section for $p p \rightarrow t \bar{t}+1$-jet $+X$. The variable $\rho_{\mathrm{s}}$ is defined as $\rho_{\mathrm{s}}=2 m_{0} / \sqrt{s_{t \bar{t} j}}$ with $m_{0}=170 \mathrm{GeV}$ and $\sqrt{s_{t \bar{t} j}}$ is the invariant mass squared of the multijet system.

\section{Results and discussions}

The differential distribution $\left(\mathrm{d} n_{3} / \mathrm{d} \rho_{\mathrm{s}}\right)\left(m_{\text {top }}^{p}, \mu, \rho_{\mathrm{s}}\right)$ was studied using NLO calculations [1] for different top quark pole masses from $m_{\text {top }}^{p}=160$ to $180 \mathrm{GeV}$. The results are shown in figure 1a. A clear separation between the mass distributions for different top quark masses is observed except in the region of $0.55<\rho_{\mathrm{s}}<0.62$ where the curves cross due to the normalization of $\left(\mathrm{d} n_{3} / \mathrm{d} \rho_{\mathrm{s}}\right)\left(m_{\mathrm{top}}^{p}, \mu, \rho_{\mathrm{s}}\right)$. As a consequence, a decrease of sensitivity is observed in the crossing region.

The impact of the scale (solid line) and the PDF choice (dashed line) on the top mass value (for $m_{\text {top }}=170 \mathrm{GeV}$ ) considered in this exercise is shown in figure $1 \mathrm{~b}$. It shows that a theoretical uncertainty of $500-600 \mathrm{MeV}$ can be reached in a mass measurement in the $\rho_{\mathrm{s}}>0.62$ interval considering the given scale and PDF choice dependences. The crossing region is again excluded due to the vanishing sensitivity. These curves were obtained by assuming a linear dependence of $n_{3}$ on the top quark mass for intervals of $\Delta m_{\mathrm{top}}^{p}=5 \mathrm{GeV}$.

\subsection{Systematic studies with POWHEG}

To further investigate the impact of higher orders and the effect of parton shower, we have compared the predictions for the $n_{3}$ distribution using different approximations: perturbative LO, POWHEG $t \bar{t}[6]$ at NLO and POWHEG $t \bar{t}+1-$ jet $+X$ [7] at NLO. The study was 

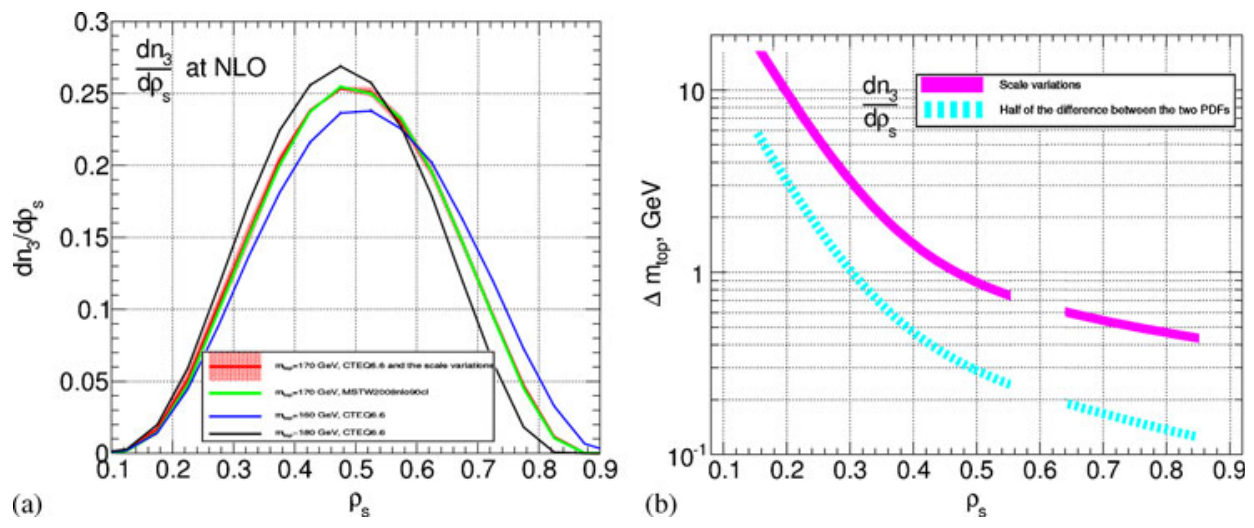

Figure 1. (a) $\mathrm{d} n_{3} / \mathrm{d} \rho_{\mathrm{s}}\left(m_{\text {top }}^{p}, \mu\right)$ calculated at NLO for different masses $m_{\text {top }}^{p}=$ 160,170 and $180 \mathrm{GeV}$. For $m_{\text {top }}^{p}=170 \mathrm{GeV}$ the scale and PDF uncertainties evaluated as discussed in the text are shown. (b) This plot indicates the impact of the scale (magenta solid line) and the PDF choices (blue dashed line) on the top mass value (for $\left.m_{\text {top }}^{p}=170 \mathrm{GeV}\right)$. The crossing region is excluded.

performed at partonic level for stable top quarks. The partonic shower was implemented using Pythia [8].

The comparison between fixed-order NLO and all these different approaches gives consistent results, especially in a large $\rho_{\mathrm{s}}$ interval $\left(\rho_{\mathrm{s}}>0.4\right)$ where small differences are seen. This shows that in general perturbative corrections are small and well under control.

\section{Conclusions and prospects}

In this article a new distribution $\mathrm{d} n_{3} / \mathrm{d} \rho_{\mathrm{s}}\left(m_{\text {top }}^{p}, \mu\right)$ is presented and its theoretical properties are studied at NLO accuracy. The distribution is shown to be sensitive to the top quark mass with well-understood theoretical behaviour with respect to the scale variations and PDF choices. Using the distribution $n_{3}$ to measure the top quark mass an uncertainty below $1 \mathrm{GeV}$ could be reached.

This study was presented to the society at the 2011 Lepton-Photon Conference for the first time as a new way to measure a top quark mass in proton-proton colliders that can be compatible and at the same time competitive with the other known methods. This is a preliminary study that will be completed in future publications. Some of the planned improvements are summarized here:

(1) Optimization of the kinematical cuts on the jet.

(2) Additional tests should be performed to evaluate the theoretical dependences, for example: consider to study the scale dependences separately for the numerator or the denominator of the $\mathrm{d} n_{3} / \mathrm{d} \rho_{\mathrm{s}}\left(m_{\text {top }}^{p}, \mu\right)$ distribution.

(3) Study the impact of more realistic/experimental situations identifying decayed top quarks and jets within an LHC detector framework. 


\section{Simone Alioli et al}

\section{References}

[1] S Dittmaier, P Uwer and S Weinzierl, Eur. Phys. J. C59, 625 (2009)

[2] P M Nadolsky et al, Phys. Rev. D78, 013004 (2008)

[3] A D Martin et al, Eur. Phys. J. C63, 189 (2009)

[4] M Cacciari and G P Salam, Phys. Lett. B641 (2006), hep-ph/0512210

[5] M Cacciari, G P Salam and G Soyez, J. High Energy Phys. 63, 0804 (2008)

[6] S Frixione, P Nason and G Ridolfi, J. High Energy Phys. 126, 0709 (2007)

[7] S Alioli, S Moch and P Uwer, J. High Energy Phys. 2012, 137 (2012)

[8] T Sjostrand, S Mrenna and P Skands, Comput. Phys. Commun. 178, 85 (2008) 